\title{
The grand challenges in smart materials research
}

\author{
Seung-Bok Choi * \\ Department of Mechanical Engineering, Inha University, Incheon, South Korea \\ *Correspondence: seungbok@inha.ac.kr \\ Edited and reviewed by: \\ Weihua Li, University of Wollongong, Australia
}

Keywords: smart materials, magnetorheological fluid, electrorheological fluid, magnetorheological elastomer, electroactive polymers

The current generation of smart materials has diverse dynamic features that enable them to adapt to the environment and make them the materials of the future. There are four of these aspects of which at least one is incorporated in a functional smart-material based device: smart materials can be sensors or actuators, they can be controlled or they can have biomimetic characteristics.

Sensors are either bonded to the surface of a structural material or are embedded within a smart material to produce an electric signal thanks to the static or dynamic changes in of the structural material. Actuators are typically excited by an external stimulus, such as electricity, in order to change the stiffness and damping properties in a controlled manner. The control capability permits the dynamic behavior of the material to respond to an external stimulus according to a prescribed control algorithm associated to microprocessors. Biomimetic characteristics are inspired by biological patterns in order to equip materials with the possibility of self-diagnosis, self-repair, and self-degradation of a broad range of structural materials.

Among materials with the above features, electrorheological (ER) fluids, magnetorheological (MR) fluids, magnetorheological (MR) elastomers, and electroactive polymers (EAPs) are in the spotlight of both the most active research activity and of the most commercial interest. For this reason, I expand and highlight grand challenges of these four smart material classes.

Electrorheological fluids (ER fluids) are smart suspensions of soft conductive particles in viscous liquid medium. ERs can be reversibly solidified from and to a free-flowing liquid state in the presence of an external electric field. This transition is associated to significant changes in the rheological characteristics of the fluid including the generation of yield stress, increased shear viscosity and elastic properties. Despite the abundant research performed on ER fluid technology over last two decades, there is no commercial ER fluid device available up to now.

In order to overcome this, the community needs to resolve to create an ER fluid that can produce high yield stress. A so-called giant electrorheological (GER) fluid, producing 50-60 times higher yield stress than conventional ER, is under development (Wen et al., 2003, 2004) involving barium titanyl oxalate nanoparticles coated with a thin urea layer (nanoparticles: $50-70 \mathrm{~nm}$, urea layer dielectric constant 60 at $10 \mathrm{~Hz}, 3-10 \mathrm{~nm}$ thick, can produce yield stress up to $130 \mathrm{kPa}$ at $5 \mathrm{kV} / \mathrm{mm}$ field strength).

Yet, unmet market needs of for ER fluids consist of the need to be non-toxic to both humans and the environment, to be chemically durable and physically stable without particle settling issues, to have low power consumption and manufacturing cost, to have a wide working temperature range covering -40 to $200^{\circ} \mathrm{C}$, to be compatible with sealing materials, to be non-abrasive and non-corrosive to the device, and to have rapid on/off responses.

Furthermore, significant research effort is invested in commercializing the ER fluid with surfactants and additives, which can potentially broaden the applicability of ER fluids while maintaining a user-friendly point of view.

Magnetorheological fluids are smart suspensions of soft magnetizable particles in non-magnetic liquid medium. Analogously to ER fluids, their phase and their rheological properties can be altered by an external magnetic field. However, contrarily to ER fluids, they are commercially applied in a broad spectrum of areas due to their field-tunability. Shock absorbers, brakes, clutches, seismic vibration dampers, control valves, and precision polishing are just a few examples to illustrate their use.

Derived from the thermal decomposition of iron pentacarbonyl, carbonyl iron (CI) particles are widely employed as a dispersed phase for MR fluids because of their outstanding magnetic properties and suitable particle size. Nevertheless, further engineering development of CI particles is impeded by sedimentation and stability problems caused by the large density difference between CI particles and the medium. Various strategies have been proposed to this issue: adding stabilizers or additives, and modifying the magnetic particles with coating technology. Dispersion stability can be improved by occupying the interspaces between magnetic particles and to prevent the physical contact of CI particles. Prevailing methods exploiting these ideas involve introducing nano/sub-micron sized fillers such as carbon nanotubes, graphite nanotubes, graphene oxide, fumed silica, glass bead, and organoclay (Powell et al., 2012).

Coating techniques provide an effective way to reduce particle density and prevent chemical oxidation of magnetic particles. Introducing suspension polymerization in the core-shell coating procedure, with CI particles as a core material, one can obtain a $2 \sim 10 \mu \mathrm{m}$ coating thickness. This is much thicker than that of the composites prepared by a dispersion polymerization, which yields about submicron coating thickness. Dual-step coating on the CI particle surface with carbon nanotubes as the final layer would not only reduce the density of the CI particles but also provide rough surfaces. The resulting composites are expected to be for improving dispersion stability while retaining MR characteristics (Park et al., 2010).

The rheological properties of MR fluids, including yield stress analysis as a 
function of magnetic field strength, are important not only because of the effect on their MR performance but also because of their further potential industrial applications. This provides a strong motivation for researchers to continuously improve them by experimenting with novel recipes. In addition, more work should be seriously considered on the chemical stability, low sedimentation, low abrasiveness, and high durability to achieve more practical applications.

Magnetorheological elastomers (MREs) are composites where magnetic particles are suspended in a non-magnetic solid or gel-like matrix. The strength of MREs is typically characterized by their fielddependent modulus. Under dynamic loading, MREs exhibit linear viscoelastic properties even at a large range of strain amplitude up to 50\% (Li et al., 2010). A number of non-parametric and parametric models have been developed to describe MREs performances. For example, a quasi-static model was proposed to explain the modulus increase by calculating the magnetic interaction between the adjacent particles. Finite element methods were also used to analyze the modulus increase under varied magnetic fields. Most models are based on the dipole model for particle energy interaction, and they rely on the assumption that the particles are the same size and shape. These results showed that the fielddependent modulus of MREs varies with the square of the saturation magnetization of the particles.

Magnetorheological elastomers are capable of sensing: MRE resistance is dependent on the deformation strain and applied magnetic field. By including graphite as additives, MREs can also show a controllable electrical resistance by adjusting the applied normal force and the magnetic field which, in turn, provides a possibility for MRE to function as a sensing material (Tian et al., 2011).

Current rubber-based MREs have a relatively high initial stiffness, which results in relatively low MR effect. The use of composites as a matrix instead of rubber in order to get an ultrasoft MR with low initial shear modulus has significantly enhanced this. The low initial shear modulus, however, weakens the support the MRE could provide, which results limiting its applications.
The interaction between the matrix base and the magnetic particles is another yetto-be-investigated challenge, with a focus on both mechanistic studies and working principles of MREs.

In the future, I expect research on softening the stiffness of MREs. With the help of a decreasing shear modulus and increasing magnetic field, one could move toward innovative engineering far beyond their current use for shock absorbers and vibration isolators. Furthermore, by making use of multifunctional MREs, it is even imaginable to fulfill MRE sensing, actuating, and damping capabilities in one package. Thus, the future trends of MRE research not only include materials innovation but, in large part, also application and device development.

Electroactive polymers are polymerbased composites in which electronic or ionic properties are embedded. EAPs exhibit a large displacement in response to external stimuli and hence can offer operational similarity to biological muscles. This is the reason why EAP materials have a really promising future in biologically inspired actuators. They may drive various mechanisms for manipulation and mobility including microrobots, micro flying objects, tactile, and animatronic devices.

Generally, the EAPs are divided into two major categories based on their activation mechanisms: electronic (driven by electric field or Coulomb forces) and ionic (involving mobility or diffusion of ions) (Bar-Cohen, 2001). The electronic polymers, such as electrostrictive, electrostatic, piezoelectric, and ferroelectric polymers require a high activation field, close to the breakdown level. In contrast, ionic EAP materials, such as gels, polymermetal composites, conductive polymers, and carbon nanotubes require low drive voltage.

Several different types of EAP materials have been developed and investigated on both material characteristics and application devices.

For example, cellulose, a renewable biopolymer has been rediscovered as an active material that can be used for sensors and actuators (Kim et al., 2006). In this context, cellulose forms electroactive paper (EAPap) and has advantages in terms of biocompatibility, biodegradability, easy modification, eco-friendliness, and low price. Cellulose EAPap has a piezoelectric effect because regenerated cellulose has a non-centro-symmetry of monoclinic crystal structure. Ions and water molecules can easily interact with its abundant hydroxyl groups, causing an ion migration effect.

Another ionic EAP, ionic polymer metal composite (IPMC) exhibits a large bending strain with low drive voltage. Strong emphasis has been put on investigating various ionic polymer membranes for highperformance IPMC actuators and overcoming some drawbacks of ionic polymer actuators to improve stability and reliability. A highly durable and waterfloatable IPMC has been reported by employing hydrophobic and asymmetrically laser-scribed reduced graphene oxide paper electrodes (Kim et al., 2014).

The next generation of EAP materials will have good deformation, stability, and efficiency. This, however, requires a bold departure from current EAP research since much of it tends to focus on the development and understanding of new polymer materials. Moreover, shortcomings of currently available EAP include the increase of actuation force, decrease of the response time, decrease of the voltage requirement, and the enhancement of the robustness under unpredictable environment.

Beyond these four smart material classes that are currently emphatic in our daily lives, shape memory alloys, piezoelectric materials, magnetostrictive materials, and electrostrictive materials are also very attractive for future studies. The next decade will bring a deeper understanding on the atomic and molecular level of our current smart materials in addition to the fundamental mechanisms associated to the intelligence of these. These two lines of research will definitely boost the discovery of novel smart materials and their innovative applications.

\section{ACKNOWLEDGMENTS}

This grand challenges article has been written based on brief reports from associate editors in Smart Materials Section of Frontiers in Materials; electrorheological fluid from Prof. W. Wen, magnetorheological fluid from Prof. H. J. Choi, magnetorheological elastomer from Prof. W. Li, and 
electroactive polymer from Prof. J. Kim. The author gratefully thanks all associate editors for providing very valuable reports on the state-of-the-art challenges based on their outstanding research specialties.

\section{REFERENCES}

Bar-Cohen, Y. (ed.) (2001). Electroactive Polymer (EAP) Actuators as Artificial muscles. Washington: SPIE Press.

Kim, J., Jeon, J.-H., Kim, H.-J., Lim, H., and Oh, I.K. (2014). Durable and water-floatable ionic polymer actuator with hydrophobic and asymmetrically laser-scribed reduced graphene oxide paper electrodes. ACS Nano 8, doi:10.1021/nn500283q

Kim, J., Yun, Y., and Ounaies, Z. (2006). Discovery of cellulose as a smart material. Macromolecules 39, 4202-4206. doi:10.1088/0957-4484/25/9/092001

Li, W. H., Zhou, Y., and Tian, T. F. (2010). Viscoelastic properties of MR elastomers under harmonic loading. Rheologica Acta 49, 733-740. doi:10.1007/ s00397-010-0446-9

Park, B. J., Fang, F. F., and Choi, H. J. (2010). Magnetorheology: materials and application. Soft Matter 6, 5246-5253. doi:10.1039/c0sm00014k

Powell, L. A., Wereley, N. M., and Ulicny, J. (2012). Magnetorheological fluids employing substitution of nonmagnetic for magnetic particles to increase yield stress. IEEE Trans. Magn. 48, 764-767. doi:10. 1109/TMAG.2012.2202885

Tian, T. F., Li, W. H., and Deng, Y. M. (2011). Study of sensing capabilities of MR elastomers. Smart Mater. Struct. 20, 025022. doi:10.1088/0964-1726/ 20/2/025022

Wen, W., Huang, X., and Sheng, P. (2004). Particle size scaling of GER. Appl. Phys. Lett. 85, 299-301. doi:10.1063/1.1772859

Wen, W., Huang, X., Yang, S., Lu, K., and Shing, P. (2003). The giant ER effect in suspensions of nanoparticles. Nat. Mater. 2, 727-730. doi:10.1038/ nmat993
Conflict of Interest Statement: The author declares that the research was conducted in the absence of any commercial or financial relationships that could be construed as a potential conflict of interest.

Received: 04 July 2014; accepted: 21 July 2014; published online: 06 August 2014.

Citation: Choi S-B (2014) The grand challenges in smart materials research. Front. Mater. 1:11. doi: 10.3389/fmats.2014.00011

This article was submitted to Smart Materials, a section of the journal Frontiers in Materials.

Copyright (c) 2014 Choi. This is an open-access article distributed under the terms of the Creative Commons Attribution License (CC BY). The use, distribution or reproduction in other forums is permitted, provided the original author(s) or licensor are credited and that the original publication in this journal is cited, in accordance with accepted academic practice. No use, distribution or reproduction is permitted which does not comply with these terms. 